\title{
Classical probability model-based new match system to badminton influence study
}

\author{
Shaowen Yan \\ Institute of Physical Education, Huanggang Normal University, Huangzhou 438000, China
}

\begin{abstract}
At present, Badminton powerhouse in the world has been monopolized by partial countries in Middle-South Asia, referencing table tennis reformation successful experience, new match system as badminton 21 -score-point has magnificent appeared. The paper utilizes classical probability model to establish single rally winning probability and single game winning probability functional relationships, by observing, applies approach of simulating image through hyperbolic tangent function curve to further simplify expression. Through comparing each evaluated objects and ideal solution as well as negative ideal solution relationships, it goes ahead with further comparison, and then gets comprehensive evaluations on four kinds of match systems. By comparing each evaluated object and ideal solution as well as negative ideal solution relationships, it gets conclusion that 21- point two out of three sets and 15-point three out of five sets are relative reasonable match systems.
\end{abstract}

\section{Introduction}

Events through reforming systems, no matter from audients to reporters, or from each country players, coaches to referees, all have different opinions on badminton 21-score-point match system. Since December.11th, 2005, international badminton association board has made decision that all go ahead with trial implementation of 21-point system match form since February.1st, 2006; The decision applied to men and women's singles, men and women's doubles as well as mixed doubles totally five items. In April, 2006 'Thomas-Uber'that held in Japan applied the new match system, which was 21-score-point new match system. International badminton federation held a conference to make decisions in Tokyo, Japan in May.6th, through all international badminton federation members' representatives votes, smoothly passed and decided to abolish 15-point match system, and meanwhile formal applied 21-score-point system. Brand new score system was initially adopted in 2008 Beijing Olympic Games. New match system let match to be more wonderful and more confrontational.

During model establishment, the paper firstly establishes four sub models those are respectively single game probability model, single match probability model, player competitive strength model and single rally probability model, according to first and second sub models establishment, and then solves relational expression between single rally wining and single game winning as well as single rally winning and single match winning probability functions, and further simplifies functions expressions. Due to match systems' differences couldn't decide that one match system has absolute advantage or disadvantage, during the all evaluation procedures, the paper applies comprehensive evaluation analysis and relativity analysis approaches to study procedures given schemes. It gets conclusion which kinds of scoring way are proper in general match system and special match system.

\section{Model establishment and solution}

\subsection{Model analysis}

Model establishment mainly takes singles as examples.

\subsection{Player competitive strength model:}

Factors that disturb a badminton player technical level performing are various that include techniques, psychological factors, strength, reaction capacity, speed and so on. And meanwhile, different players have different characteristics of confrontational strength in service, return of the service, front court and back court as well as other links, if it really needs to measure every factor, it will cause certain difficulties in creating indicators that comprehensive evaluate players' competitive strength. It is difficult to make comprehensive evaluation of players' competitive levels so as to weigh each factor. But to further realize model simplification effects, and meanwhile don't affect model effectiveness, on such premise, we fully can suppose that player's technical level can use standardized indicator $\mu$ to judge its standard. In the paper, it defines

a Corresponding author: ShaowenYan@126.com 
$\mu$ as player original technical level, when and only when $\mu_{1}>\mu_{2}$, it is supposed that player a technical level is better than that of player $b$.

Meanwhile, in competitions it also should consider any one non-technical factor that causes different results. According to above various causes, player performed levels in competition process are closely linked to many kinds of causes, and these causes all belong to random variables, precise modeling process relative difficult aspects. According to Independent identically distributed central limit theorem, assume that player single rally competition on spot competitive capacity as random variable $\mathrm{X}$, and then it has $X \sim N\left(\mu, \sigma^{2}\right)$

From which ${ }^{\mu}$ represents players' original technical level (performed average technical level at normal state at ordinary times), $\sigma$ represents player state performing stability (deviation degree between in-situ competition level performing and original level performing).Symbol description is as Table 1 .

\subsection{Single rally probability model}

Assume player a and $\mathrm{b}$ during one rally competition, player a spot performance level arrives at X1, player b spot performance level arrives at $\mathrm{X} 2$, player a winning probability in the rally is $P\left\{X_{1}>X_{2}\right\}$.Due to basic assumption without considering mutual interference among players, then it is thought that random variables $\mathrm{X} 1, \mathrm{X} 2$, are independently identically distributed. Since $X_{1} \sim N\left(\mu_{1}, \sigma_{1}^{2}\right), X_{2} \sim N\left(\mu_{2}, \sigma_{2}^{2}\right)$, so that $\mathrm{X}_{1}$ probability density function is: $f_{1}(x)=\frac{1}{\sqrt{2 \pi}} \exp \left(-\frac{\left(x-\mu_{1}\right)^{2}}{2 \sigma_{1}{ }^{2}}\right) \quad X_{2}$ probability density function is: $f_{2}(x)=\frac{1}{\sqrt{2 \pi}} \exp \left(-\frac{\left(x-\mu_{2}\right)^{2}}{2 \sigma_{2}{ }^{2}}\right)$

$$
\begin{aligned}
X_{1}, & X_{2} \text { joint probability density function } \\
\text { is: } & f(x, y)=\frac{1}{2 \pi \sigma_{1} \sigma_{2}} \exp \left(-\frac{\left(x-\mu_{1}\right)^{2}}{2 \sigma_{1}^{2}}-\frac{\left(x-\mu_{2}\right)^{2}}{2 \sigma_{2}^{2}}\right)
\end{aligned}
$$

Player a winning probability in the single rally competition is:

$$
p=\iint_{x>y} \frac{1}{2 \pi \sigma_{1} \sigma_{2}} \exp \left(-\frac{\left(x-\mu_{1}\right)^{2}}{2 \sigma_{1}{ }^{2}}-\frac{\left(x-\mu_{2}\right)^{2}}{2 \sigma_{2}{ }^{2}}\right) d x d y
$$

And then player $\mathrm{b}$ winning probability in the single rally competition is $q=1-p$.

\subsection{Single game probability model}

The paper can utilize classical probability theory approach to calculate player $a$ and $b$ single game winning probabilities. According to original badminton match system backgrounds conditions, it can calculate no follow occurrence probability and follow occurrence probability.

It can base on player a winning status as examples to discuss (The analysis would not affect question's essence, because a, b have symmetry here, so we only compare a winning status).

(i) In case that draw $(i-1):(i-1)$ not held between two parties, player a won by achieving $i$ balls, then the rally ended. Through analysis we know $P\left(A_{i}\right)=\sum_{n=i}^{\infty} A_{n}$

Every rally can be regarded as Bernoulli experiment, then $P(A n)=C_{n-1}^{i-1} p^{i-1} q^{n-i} p=C_{n-1}^{i-1} p^{1} q^{n-i} \quad(n=i+k, k=0,1,2, \cdots, i-2)$

(ii) In case that two parties held draw $(i-1):(i-1)$, then the two continue to play until player a got 2 scores more than $b$ so that a won the game.

From above analysis, it is known that total $\mathrm{n}$ balls were played in competition at this time, $n=2(i-1)+2 m$, from which $m=1,2,3 \cdots$

For the question, it can be analyzed in 3 stages, the first stage is previous $2(\mathrm{i}-1)$ balls, player $a, b$ each win $\mathrm{i}-1$; in the second stage in order to play $2 m$ follows, from which every two rallies can be regarded as one round; In each round of previous $\mathrm{m}-1$ rounds, player $\mathrm{a}, \mathrm{b}$, each won 1 ball, while in $\mathrm{m}$ round of last round, player a won the game as long as continuously won two balls that is:

\begin{tabular}{|c|c|}
\hline$i$ & Badminton match \\
\hline$h$ & It represents match system, generally is $h$ out of $(2 h-1)$ sets. \\
\hline$(i, h)$ & It represents match system is $i$-point system, adopts $h$ out of $(2 h-1)$ sets \\
\hline$A_{n}$ & $\begin{array}{c}\text { It represents in case } i \text {-point system, after playing } n \text { rallies, one game ends and } a \\
\text { wins“ }\end{array}$ \\
\hline$f_{i}(p)$ & It represents player $a$ single set winning probability in score system $i$ \\
\hline$g(x)$ & It represents $f_{i}(p)$ curve fitting function \\
\hline$\varphi_{i}(2 h-1, h, p)$ & $\begin{array}{l}\text { It represents player } a \text { single match winning probability in score system } i, \\
\text { game }(2 h-1) .\end{array}$ \\
\hline$g\left(p, \beta_{h}^{(i)}\right)$ & It represents $\varphi_{i}(2 h-1, h)$ curve fitting function. \\
\hline$O C(i, h)$ & It represents contingency index in score system $i$, game $(2 h-1)$. \\
\hline$\Omega_{h}^{(i)}(p)$ & It represents intensity index function in score system $i$, game $(2 h-1)$. \\
\hline
\end{tabular}

$$
P^{\prime}(A n)=C_{2(i-1)}^{i-1} p^{i-1} q^{i-1}(2 p q)^{m-1} p^{2}=C_{2(i-1)}^{i-1} p^{i+1} q^{i-1}(2 p q)^{m-1}
$$

Table 1. Symbol description. 
So in case score system is $i$, every match a winning probability is :

$$
\begin{aligned}
f_{i}(p) & =\sum_{k=0}^{i-2} C_{i+K-1}^{i-1} P^{i} q^{k}+\sum_{m=1}^{\infty} C_{2(i-1)}^{i-1} P^{i+1} q^{i-1}(2 p q)^{m-1} \\
& =p^{i} \sum_{k=0}^{i-2} C_{i+K-1}^{i-1} q^{k}+C_{2(i-1)}^{i-1} P^{i+1} q^{i-1} \frac{1}{1-2 p q}
\end{aligned}
$$

Use MATLAB simulation software to draw corresponding single rally winning probability $p$ and single game winning probability $f_{i}(p)$ relational figure(Figure 1), here add the definition of $i$, define $i=7,11,17,21,27$.Figure 1 is relation figure between single rally winning probability $p$ and single game probability $f_{i}(p)$ when $i=7$.

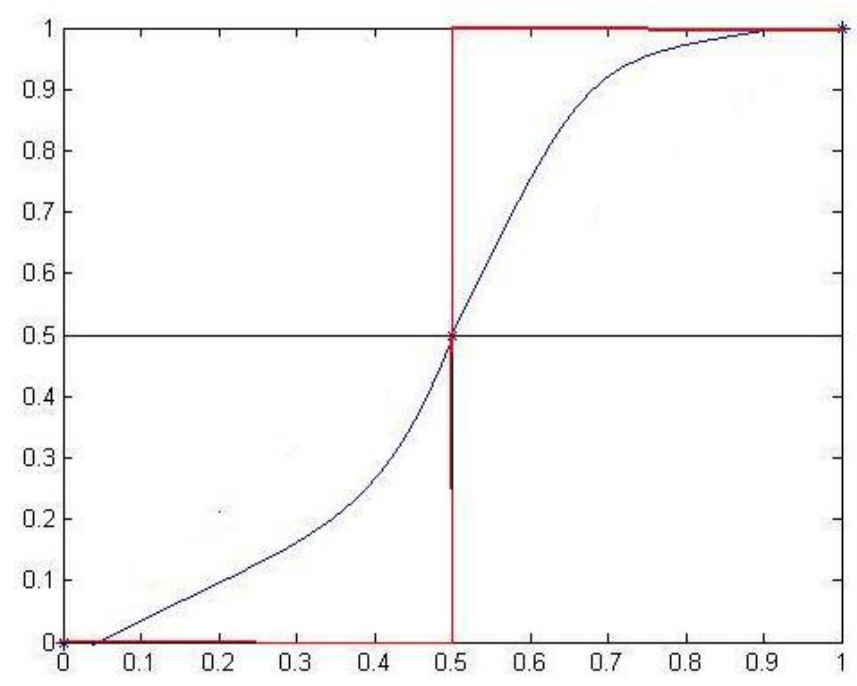

Figure 1. 7 points system single rally winning probability and single game probability functional relations.

In Figure 1, function line successively represents 7 points system single rally winning probability and single game probability functional relations. Similarly, it is supposed that single rally winning probability shows player comprehensive competitive strength under $i=15,17,21,27$, due to certain occasional time would hard to avoid appearing in competition, then it is supposed when single game winning probability completely is unrelated to single rally winning probability that no matter what two parties players competitive strength would be, the winning probability is always 0.5 , the competition result is completely accidental; when single game winning probability entirely depends on single rally winning probability that when higher competitive strength player would surely win, then competition result is completely non-accidental(as Figure 1); when competition rules changed score system gets lower, curve then gets closer to entirely accidental function line. It is clear from figure that curve slope reflects contingency occurs under different score systems, from 7 points system to 27 points system, each curve slope $\alpha$ successively increases, while contingency successively deceasing so that it can be found that negative correlation existing between slope $\alpha$ and contingency. Above Figure 1 defined $x=0.5$ and $y=0.5$ are two asymptotic lines, when $\alpha$ changes from 0 to $\infty$, curve sweeps $[0,0.5] *[0,0.5]$ and $[0.5,1] *[0.5,1]$ two areas, in subsequently models, it just use $\alpha$ to reflect different competition systems' contingency indicators that brings great convenience to calculation.

\subsection{Single match probability model}

Badminton match normally adopts competition law of $h$ out of $2 h-1$ sets , assume that player $a, b$ in $2 h-1$ games competition, each game competition is independent, which is also giving player $a$ the same winning probability as $f_{i}(p)$. Given event $B(s)$ to be $b$ totally won $s$ games, and finally player $a$ won, then it has: $P\left(B((s))=C_{h+s-1}^{s}\left(1-f_{i}(p)\right)^{s} f_{i}^{h}(p)\right.$

Given event $B$ to be " $a$ won the competition", then it has: $P(B)=\sum_{s=0}^{h-1} P\left(B((s))=f_{i}^{h}(p) \sum_{s=0}^{h-1} C_{h+s-1}^{s}\left(1-f_{i}(p)\right)^{s}\right.$

Therefore we have expressions as following:

$$
\varphi_{i}(2 h-1, h, p)=f_{i}^{h}(p) \sum_{s=0}^{h-1} C_{h+s-1}^{s}\left(1-f_{i}(p)\right)^{s}
$$

According to above shown computational methods, single match winning probability is the function of single game winning probability, therefore it is also the function of single rally winning probability, through MATLAB simulated figure, it is clear that 4 schemes single match winning probability and single rally winning probability correlations functional image, through observation of functional relationships graphics, it is found that graphics all go through fixed point $(0.5,0.5)$, the graphics is very similar to hyperbolic tangent function figure, so it adopts hyperbolic tangent function to carry out fitting curve analysis here.

Through formula (1), formula (2), we can calculate and learn that if it directly outputs single match winning probability and single rally winning probability functional relation expression according to compound functional relations, the expression surely is very complicated, so utilizes curve fitting can let complicated polynomial to fully approximate to simple hyperbolic tangent function.

Here, we introduce fitting function $\mathrm{g}(\mathrm{x})$ that is:

$$
g(x)=\frac{2}{1+\exp (-2 \alpha(x-0.5))}-0.5
$$

Through fitting functions, it can make approximate calculation on $\varphi_{i}=(2 h-1, h, p)$ as following:

$$
\varphi_{i}(2 h-1, h, p) \approx g\left(p, \alpha_{h}{ }^{(i)}\right)=\frac{2}{1+\exp \left(-2 \alpha_{h}{ }^{(i)}(p-0.5)\right)}-0.5
$$

Among them $\alpha_{h}{ }^{(i)}$ is corresponding achieved corresponding fitting function slope when new match system is $(i, h)$.

Through MATLAB linear fitting principle, it gets inclination $\alpha_{h}{ }^{(i)}$ in 4 cases, specific numerical values status is as Table 2 . 
Table 2. 4 kinds of competition system plans inclination.

\begin{tabular}{cc}
\hline Match system & Inclination $\alpha_{h}^{(i)}$ \\
\hline 15 points three out of five sets & 4.1481 \\
21 points two out of three sets & 4.2938 \\
15 points four out of seven sets & 4.2970 \\
21 points three out of five sets & 4.4600 \\
\hline
\end{tabular}

In fitting process, in order, used value $p$ range is $(0.3,07)$ for the purpose of improving curve fitting precise, such fitting method through elimination increases fitting curve consistency to a certain basis; According to formal competition result analysis, it gets fitting curve variable $p$ range as $(0.3,07)$, when $p \in(0,0.3)$, function value is always 0 , when $p \in(0.7,1)$, function value is always 1 .

By above analysis, it can absolutely make below approximate to $\varphi_{i}(2 h-1, h, p)$, furthermore summarization is as following formula:

$$
\varphi_{i}(2 h-1, h, p)= \begin{cases}0 & , \quad(0<p<0.3) \\ \frac{2}{1+\exp \left(-2 \alpha_{h}^{(i)}(p-0.5)\right)}-0.5 & ,(0.3<p<0.7) \\ 1 & ,(0.7<p<1)\end{cases}
$$

\subsection{Evaluation indicator model}

The model is just targeted on side-out scoring system influences on total competition time, assuming other conditions are the same. According to $A, B$ two people whole competition each rally data, make statistics and get probability of continuously achieving $i$ scores.

Make statistical summary on player $A$ whole competition data; get the proportion that player $A$ continuously achieving $i$ balls of total scores: $P_{i}(A) \quad(i=1,2,3,4 \ldots)$

Determine above formula sum according to below formula, get before reform $N_{A}^{\prime}$ and after reform $N_{A}$ total score probability:

$$
N_{A}=\sum_{i=1}^{n} i \cdot P_{i}(A) \quad N_{A}^{\prime}=\sum_{i=1}^{n}(i-1) \cdot P_{i}(A)
$$

Proportional relations between established total scores probability and competition time consumption before and after reform is:

$$
\frac{h_{A}^{\prime}}{h_{A}}=\frac{N_{A}}{N_{A}^{\prime}}
$$

It gets total competition time consumption before reform under same circumstance:

$$
h_{A}^{\prime}=\frac{N_{A}}{N_{A}^{\prime}} \cdot h_{A}
$$

Make statistical summary on player $B$ whole competition data, and get the proportion that player B continuously achieving $i$ balls of total scores as:

$$
P_{i}(B) \quad(i=1,2,3,4 \ldots)
$$

Determine above formula sum according to below formula, get before reform $N_{B}{ }^{\prime}$ and after reform $N_{B}$ total score probability:

$$
N_{B}=\sum_{i=1}^{n} i \cdot P_{i}(B) \quad N_{B}^{\prime}=\sum_{i=1}^{n}(i-1) \cdot P_{i}(B)
$$

Proportional relations between established total scores probability and competition time consumption before and after reform is:

$$
\frac{h_{B}^{\prime}}{h_{B}}=\frac{N_{B}}{N_{B}^{\prime}}
$$

It gets total competition time consumption before reform under same circumstance:

$$
h_{B}^{\prime}=\frac{N_{B}}{N_{B}^{\prime}} \cdot h_{B}
$$

Due to make respective data statistics among opponents, their data have a certain correlation, determine the two average values and finally get total competition time consumption before reform as:

$$
h=\frac{h_{A}{ }^{\prime}+h_{B}{ }^{\prime}}{2}
$$

In order to better verify before and after side-out scoring system cancelling effects on total competition time consumption, it takes badminton men singles final $\mathrm{Li}$ Zhong-Wei verse Lin Dan data in London Olympic Games, 2012 as an example like following Table 3.

Utilize fixed time model indicator model to make statistics of each rally scores and calculate as following Table 4.

\subsection{Match system schemes evaluation}

Match has been transformed form original '15-point system to 21-point system. It is able to reduce players' injuries to a great extent, correspondingly can also increase players' positivity and improve television relay quality, and meanwhile it is clear through the model that match total time consumption shortens by match system reforming, in the way each event badminton committee can leisurely arrange matches, and audients can also watch more and wonderful badminton matches. Besides, new match system also puts forward higher requests on referees; the referees' penalty accuracy per time would decide players' scores. The control of penalty scale by referees should be 
further improved; the new match system creates conditions for badminton match booming.

Table 3. Men single final scores statistics under new match system on 2012.08.05.

\begin{tabular}{|c|c|c|c|c|c|}
\hline \multicolumn{2}{|c|}{ The first game } & \multicolumn{2}{|c|}{ The second game } & \multicolumn{2}{|c|}{ The third game } \\
\hline 1: & 0 & $0:$ & 1 & 1: & 0 \\
\hline 1: & 1 & 1: & 1 & 1: & 1 \\
\hline 1: & 2 & 1: & 2 & $2:$ & 1 \\
\hline 2: & 2 & 1: & 3 & $2:$ & 2 \\
\hline 3: & 2 & $2:$ & 3 & $2:$ & 3 \\
\hline 3: & 3 & 3: & 3 & 3: & 3 \\
\hline 4: & 3 & 4: & 3 & 3: & 4 \\
\hline 5: & 3 & 4: & 4 & 4: & 4 \\
\hline 5: & 4 & 4: & 5 & 5: & 4 \\
\hline 5: & 5 & 5: & 5 & 5: & 5 \\
\hline 5: & 6 & 5: & 6 & $6:$ & 5 \\
\hline 6: & 6 & $6:$ & 6 & $7:$ & 5 \\
\hline 7: & 6 & $6:$ & 7 & 8: & 5 \\
\hline 7: & 7 & $6:$ & 8 & 8: & 6 \\
\hline 8: & 7 & $7:$ & 8 & 8: & 7 \\
\hline 8: & 8 & $7:$ & 9 & 8: & 8 \\
\hline 9: & 8 & $7:$ & 10 & 8: & 9 \\
\hline 10: & 8 & 7: & 11 & 9: & 9 \\
\hline 11: & 9 & 7: & 12 & 9: & 10 \\
\hline 12: & 9 & $7:$ & 13 & 9: & 11 \\
\hline 12: & 10 & 8: & 13 & 10: & 11 \\
\hline 13: & 10 & 8: & 14 & 10: & 12 \\
\hline 13: & 11 & 8: & 15 & 11: & 12 \\
\hline 14: & 11 & 8: & 16 & 12: & 12 \\
\hline 15: & 11 & 8: & 17 & 12: & 13 \\
\hline 16: & 11 & 9: & 17 & 13: & 13 \\
\hline 16: & 12 & 9: & 18 & 14: & 13 \\
\hline 17: & 12 & 9: & 18 & 15: & 13 \\
\hline 17: & 13 & 10: & 19 & 15: & 14 \\
\hline 18: & 13 & 10: & 20 & 15: & 15 \\
\hline 19: & 13 & 10: & 21 & 16: & 15 \\
\hline 19: & 14 & & & 16: & 16 \\
\hline 19: & 15 & & & 17: & 16 \\
\hline 20: & 15 & & & 18: & 16 \\
\hline 21: & 15 & & & 18: & 17 \\
\hline 21: & 15 & & & 18: & 18 \\
\hline & & & & 19: & 18 \\
\hline & & & & 19: & 19 \\
\hline & & & & 19: & 20 \\
\hline & & & & 19: & 21 \\
\hline
\end{tabular}

Table 4. Each rally scores statistics.

\begin{tabular}{cccccc}
\hline Scores & Li Zhong-Wei & Lin Dan & Scores & Li Zhong-Wei & Lin Dan \\
\hline 1 & 0.34 & 0.4 & 0 & 0.34 & 0.4 \\
2 & 0.28 & 0.32 & 1 & 0.28 & 0.32 \\
3 & 0.3 & 0.18 & 2 & 0.3 & 0.18 \\
4 & 0.08 & 0.24 & 3 & 0.08 & 0.24 \\
& 2.12 & 2.54 & & 1.12 & 1.4 \\
\hline
\end{tabular}

Total time consumption after reform:1.5(hour) Total time consumption before reform:2.780(hour)

\section{References}

1. J.L. Chang, Journal Of Chengdu Physical Education Institute 32 (2006).

2. F.G. Jiang, Journal Of Capital College Of Physical Education 17 (2005).
3. B.L. Kang, Journal Of Guilin Institute Of Tourism 4 (2011).

4. W.W. Li, H.M. Wang, China Industrial Economy 4 (2002).

5. H. Liu, W. Mi, N. Zhao, Journal Of Coastal Research 73 (2015).

6. H. Huang, Z.L. Liu Study on The Stochastic And Self-Learning Technology.

7. Ebm 2010: International Conference on Engineering and Business Management 1-8 (2010). 